EXTENDED REPORT

\title{
Immunosuppressive treatment of chronic periaortitis: a retrospective study of 20 patients with chronic periaortitis and a review of the literature
}

\author{
K Warnatz, A G Keskin, M Uhl, C Scholz, A Katzenwadel, P Vaith, H H Peter, U A Walker
}

Ann Rheum Dis 2005;64:828-833. doi: 10.1136/ard.2004.029793

See end of article for authors' affiliations

.....................

Correspondence to:

Dr K Warnatz, Department

of Rheumatology and

Clinical Immunology,

University Hospital

Freiburg, Hugstetterstr 55,

D-79106 Freiburg,

Germany; warnatz@

med1.ukl.uni-freiburg.de

Accepted

9 November 2004
Background: Retroperitoneal fibrosis (RPF) and inflammatory aneurysm of the abdominal aorta (IAAA) are regarded as two manifestations of the same disease, termed "chronic periaortitis".

Objective: To determine the optimal therapeutic and diagnostic approaches to IAAA.

Methods: The outcome of medical immunosuppressive and surgical treatment of 20 patients was examined. Measurements of the $\mathrm{C}$ reactive protein (CRP) were compared with contrast enhanced imaging studies in the follow up of the patients.

Results: The diameter of the periaortic mantle and its contrast enhancement improved in 13/15 (87\%) patients given immunosuppressive treatment for a period of more than 6 months. Strong contrast enhancement was associated with a substantial rise in CRP, but no correlation between the CRP value and thickness of the fibrotic mass was found, even at intraindividual follow up.

Conclusions: Immunosuppressive treatment should be included in the first line treatment of patients with RPF and should be maintained long term. Imaging studies are better than CRP measurements in the evaluation of response to treatment.
O rmond wrote the first English report of idiopathic retroperitoneal fibrosis (RPF) in $1948,{ }^{1}$ describing a bilateral ureteral obstruction due to compression by an inflammatory process. In 1972, Walker et al reported aortic aneurysms with an inflamed and thickened aortic wall, extensive periaortal and RPF. ${ }^{2}$ More than a decade later, Mitchinson discovered that the inflammatory aneurysms of the abdominal aorta (IAAA) differ from Ormond's disease only in the diameter of the inflamed aorta, ${ }^{3}$ and suggested that both syndromes represent only variations of the same disease, which he named "chronic periaortitis". The diagnosis is usually made by either computed tomography (CT) or magnetic resonance imaging (MRI) of the abdomen; the need for a confirmatory biopsy is controversial. ${ }^{4}$

Several observations suggest that the immune system has an important role in the pathogenesis of periaortitis. The media and adventitia of the aorta, as well as the periaortal tissue are infiltrated by polyclonal B lymphocytes, activated CD4 positive $\mathrm{T}$ lymphocytes, and plasma cells. ${ }^{5}$ In $85 \%$ of patients the necrotic medium contains deposits of IgG antibodies in close proximity to extracellular ceroid, and the serum often contains antibodies against ceroid and oxidised low density lipoprotein. ${ }^{6}$ Numerous case reports have described associations between chronic periaortitis and autoimmune diseases. ${ }^{7}$ The therapeutic strategy to relieve urinary tract obstruction is still empirical and consists of surgical interventions with intraperitoneal displacement, omentum or silicon wraps. On the other hand, several studies have demonstrated an excellent benefit of immunosuppressive treatment with and without surgical intervention. However, the optimal immunosuppressive agent, the length of treatment needed, and the best approach to monitoring disease activity have not been analysed.

We therefore retrospectively analysed 20 patients with chronic periaortitis, presenting either as RPF or as IAAA, and studied the initial symptoms, the response to immunosuppression, and the value of $C$ reactive protein (CRP) measurement or imaging studies in disease monitoring.

\section{PATIENTS AND METHODS}

The electronic patient records of our hospital were searched for the following keywords: "Ormond's disease", "retroperitoneal fibrosis", or "inflammatory aneurysm of the abdominal aorta".

Data were collected retrospectively from patient charts, a questionnaire, and by direct questioning when possible. The following information was obtained: symptoms, laboratory measurements (white blood cell count, CRP, creatinine), the findings in imaging studies at the time of diagnosis and at follow up, as well as the prior medical history. MRI was performed in 12 patients with a Siemens Sonata $1.5 \mathrm{~T}$ (Erlangen, Germany) using standard body coil or body array coils running the following sequences: spin echo $\mathrm{T}_{1}$ weighted, transversal slices before and after administration of a contrast medium $(0.1 \mathrm{mmol} / \mathrm{kg}$ gadolinium-DTPA; Magnevist, Schering AG, Berlin), slice thickness $8 \mathrm{~mm}$; fast spin echo $\mathrm{T}_{2}$ weighted, transversal and sagittal slices, slice thickness $8 \mathrm{~mm}$, as well as fat suppressed, $\mathrm{T}_{2}$ weighted inversion recovery sequence, slice thickness $10 \mathrm{~mm}$, transversal slices.

Alternatively, a spiral multislice CT scanner (Volume Zoom; Siemens, Erlangen) was the standard machine for scanning the abdomen in eight patients. Reconstructed slice thickness of the transversal slices was $5 \mathrm{~mm}$. In all patients, 100-120 ml contrast medium (Imeron; Altana AG, Konstanz) was injected intravenously during the scan procedure to obtain a good vessel contrast. The thickness of the periaortic mantle, its longitudinal extension, and contrast enhancement were scored by a single experienced radiologist, who was unaware of the temporal sequence of the images. Partial

Abbreviations: AZA, azathioprine; CRP, $C$ reactive protein; $C T$, computed tomography; CYC, cyclophosphamide; DJ, double J; IAAA, inflammatory aneurysm of the abdominal aorta; MMF, mycophenolate mofetil; MRI, magnetic resonance imaging; OCS, oral corticosteroids; $\mathrm{RPF}$, retroperitoneal fibrosis 


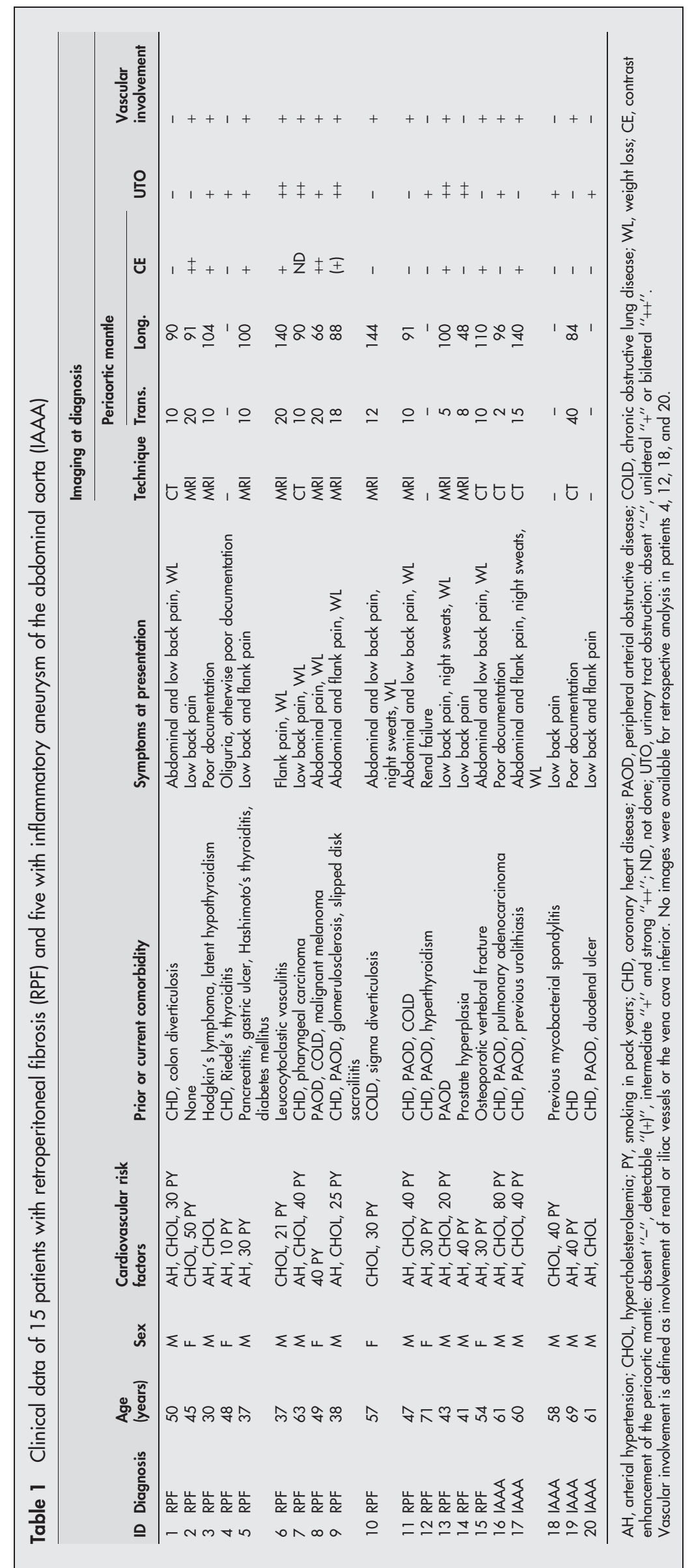



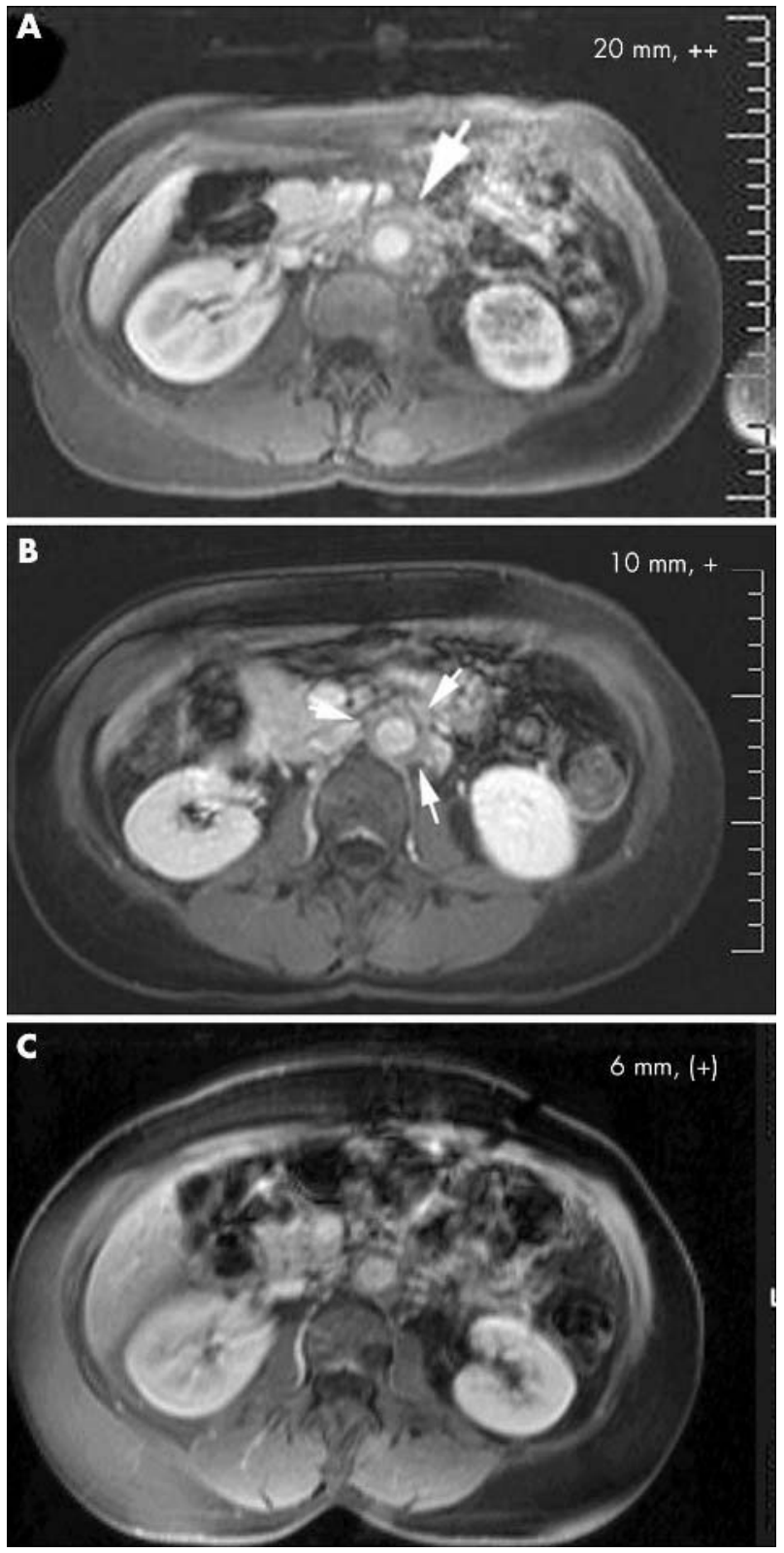

Figure 1 Serial MRI study of RPF during immunosuppressive treatment. $\mathrm{T}_{1}$ weighted images of patient 2 after (A) 2, (B) 14, and (C) 26 months of immunosuppression, indicating the diameter of the periaortic mantle and gadolinium enhancement (absent " - ", detectable " $(+)$ ", intermediate "+" and strong "++").

remission was defined as improvement, and full remission as normalisation of the respective variable.

For statistical calculation of the association between the increase in CRP and contrast enhancement, Kruskal-Wallis one way analysis of variation on ranks was used with subsequent multiple comparisons.

\section{RESULTS}

\section{Demographics and medical history}

Twenty two patients with chronic periaortitis were identified during the past 12 years and were seen by the department for urology, vascular surgery, nephrology, or rheumatology. The diagnosis of chronic periaortitis was confirmed in 20 patients (14 male, 6 female patients; table 1), based on either histology (12 patients) and/or typical radiological findings.

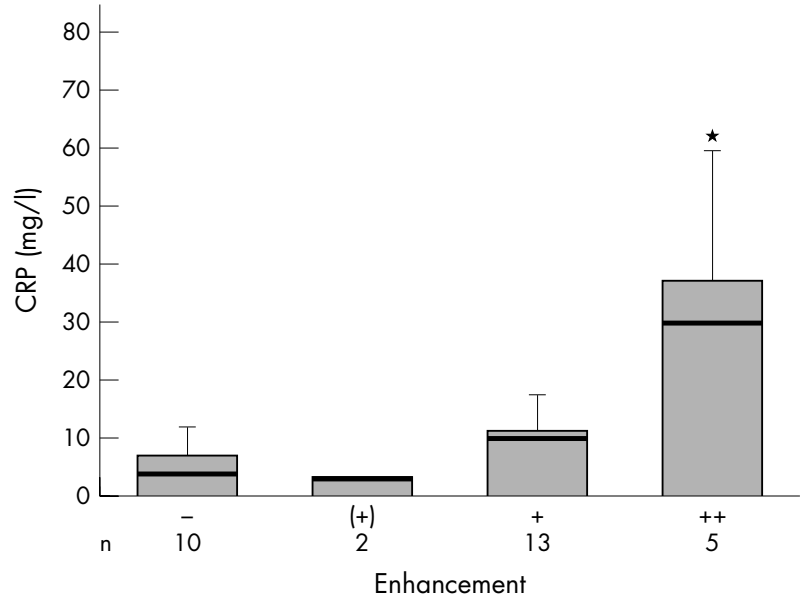

Figure 2 Association of CRP values with the intensity of contrast enhancement. Significant increases $\left({ }^{*} p=0.008\right)$ of CRP were detectable only with concomitant strong $\left({ }^{\prime \prime}++^{\prime \prime}\right)$ contrast enhancement. $n=$ number of images. Bars represent means (SD). CRP medians are represented by the thick line within each bar.

Two patients were excluded from further analysis owing to retroperitoneal dissemination of malignant cells (cholangiocarcinoma and bladder cancer) with survival of less than 6 months after diagnosis.

The average age at the time of diagnosis of chronic periaortitis was 51 years (range 30-71). Fifteen patients manifested with isolated RPF and five with IAAA. The median ages of the patients presenting with RPF and with IAAA were 49 years (range 30-71) and 62 years (range 5869), respectively. The average time of follow up was 39 months (range 5-75). Table 1 gives the medical history of the patients studied. Cardiovascular risk factors were common both in patients with IAAA and those with RPF. Fifteen $(75 \%)$ patients had a history of arterial hypertension, $13(65 \%)$ presented with hypercholesterolemia, and $18(90 \%)$ were heavy cigarette smokers, with an average of 35 pack years (range 10-80). Coronary heart disease was diagnosed in $10(50 \%)$ patients and peripheral arterial obstructive disease in $8(40 \%)$. Autoimmune disorders were recorded in $4(20 \%)$ patients and included thyroiditis, leucocytoclastic vasculitis, and sacroiliitis.

\section{Symptoms and laboratory findings at the initial presentation}

Fifteen of $16(94 \%)$ patients were referred with pain. In $11 / 16(69 \%)$ patients, the pain was localised in the lower back, in $7 / 16(44 \%)$ in the abdomen, and in 5/16 (31\%) in the flanks. Weight loss was documented in 9/16 (56\%) patients, either before diagnosis, or during the further course of the disease. One patient presented with renal failure, and two with Leriche's syndrome. Laboratory evidence of renal insufficiency (diagnosed as serum creatinine $>97 \mu \mathrm{mol} / \mathrm{l}$ at two consecutive visits) was detectable in $40 \%$ of patients. Microhaematuria was present in $30 \%$ of subjects. The CRP (normal range $<5 \mathrm{mg} / \mathrm{l}$ ) was initially increased in $10 / 13(77 \%)$ subjects and the erythrocyte sedimentation rate (Westergren's method) was $>20 \mathrm{~mm} / \mathrm{lst}$ h in $14 / 16(87.5 \%)$. Only $10 \%$ of patients presented with leucocytosis.

\section{Imaging}

Initial ultrasound examination disclosed signs of urinary tract obstruction in $13(65 \%)$ subjects (five bilateral and eight unilateral). MRI or contrast enhanced CT scans typically showed a fibrotic mass surrounding the infrarenal aorta, but 
Table 2 Therapeutic interventions and outcome

\begin{tabular}{|c|c|c|c|c|c|c|c|}
\hline \multirow[b]{2}{*}{ ID } & \multicolumn{2}{|l|}{ Treatment } & \multirow{2}{*}{$\begin{array}{l}\text { Follow up } \\
\text { (months) }\end{array}$} & \multicolumn{4}{|l|}{ Outcome } \\
\hline & Procedures & Immunosuppression & & Creatinine & CRP & DJ catheter & Mantle \\
\hline 1 & None & $\mathrm{CYC}$ & 10 & Normal & PR & - & PR \\
\hline 2 & None & CYC, AZA & 33 & Normal & FR & - & PR \\
\hline 3 & DJ & CYC, AZA & 20 & FR & Normal & Removed & $\mathrm{FR}$ \\
\hline 4 & Ureterolysis, DJ & None & 8 & PR & ND & Removed & ND \\
\hline 5 & Ureterolysis, DJ & OCS & 72 & FR & Normal & Maintained & ND \\
\hline 6 & DJ & MMF, CYC, AZA, CSA & $4 \overline{5}$ & FR & FR & Removed & FR \\
\hline 7 & DJ & AZA & 42 & PR & PR & Maintained & PR \\
\hline 8 & None & CYC & 5 & Normal & FR & - & PR \\
\hline 9 & Ureterolysis, DJ & MMF & 51 & EX & PR & Maintained & PR \\
\hline 10 & DJ & CYC, OCS & 45 & Normal & FR & Maintained & PR \\
\hline 11 & None & CYC, OCS & 40 & Normal & $\mathrm{FR}$ & - & PR \\
\hline 12 & Ureterolysis, DJ & None & 36 & PR & Ex & Removed & ND \\
\hline 13 & DJ & CSA, AZA, MTX & 42 & FR & Normal & Removed & PR \\
\hline 14 & Ureterolysis, DJ & AZA, OCS & 38 & PR & Normal & Removed & PR \\
\hline 15 & None & CYC & 9 & Normal & PR & - & PR \\
\hline 16 & Aortic graft & None & 10 & Normal & ND & - & ND \\
\hline 17 & Intraluminal aortic stent, DJ & None & 45 & PR & Normal & Maintained & EX \\
\hline 18 & Ureterolysis, DJ & AZA, OCS & 25 & FR & Normal & Removed & ND \\
\hline 19 & Left renal artery stent, DJ & OCS, CYC, AZA & 75 & PR & Normal & Maintained & PR \\
\hline 20 & Nephrectomy, aortic graft, DJ & None & 70 & EX & FR & Maintained & ND \\
\hline
\end{tabular}

excluding its posterior wall (fig 1). The average (SD) thickness and longitudinal extension of the periaortic mantle were $14.3 \quad(8.5) \mathrm{mm}$ and 99.8 (25) $\mathrm{mm}$, respectively. Involvement of the renal arteries was detected in 38\% of images and compression of the vena cava in $75 \%$ (without corresponding clinical symptoms). Contrast enhancement of the lesion was present in only 56\% of the initial images. Radiological follow up was not possible in six patients, either because only one imaging session was performed $(n=2)$ or because images could not be retrieved $(n=4)$. No correlation was found between the CRP value and the thickness of the fibrotic mass, even at intraindividual follow up (data not shown). Strong enhancement of gadolinium in the lesions was associated with high CRP values, whereas no such association was detected between slight or absent enhancement and CRP (fig 2).

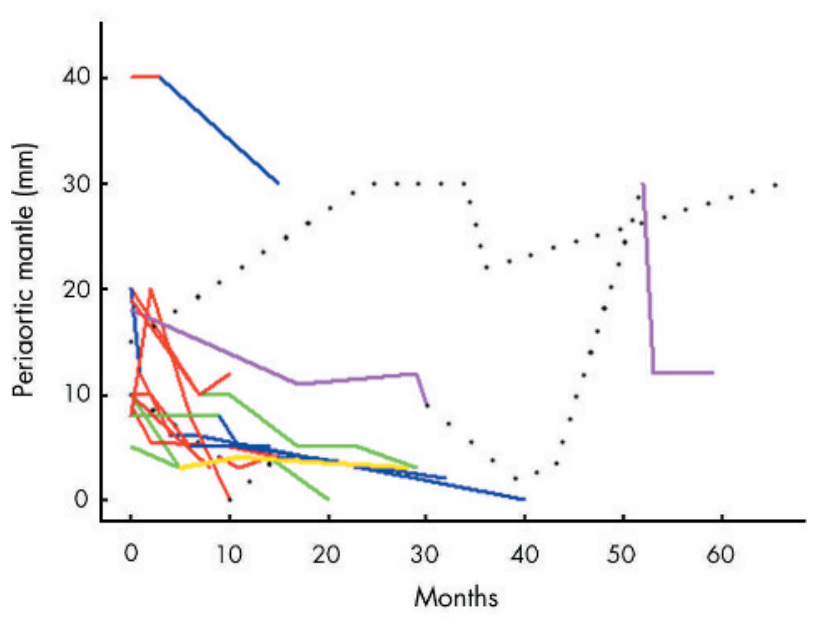

Figure 3 Evolution of the periaortic mantle (thickness in $\mathrm{mm}$ ) after the initiation of immunosuppressive treatment. Colours represent: AZA, green; CYC, red; MMF, purple; MTX, yellow; OCS, blue; no immunosuppression, black dots.

\section{Treatment}

Treatment consisted of surgical intervention without immunosuppression (5 patients), immunosuppression only (11 patients), or a combination of both (4 patients) (table 2 ). Ureteral obstruction was relieved by intraureteral stenting with a double J catheter (DJ).

Immunosuppressive drugs were used in 15 patients and reduced the diameter of the periaortic mantle in 13 (87\%) of them (figs 1 and 3). This reduction took many months of treatment in most patients (fig 3 ). Notably, $50 \%$ of the initial 14 DJ were permanently removed during immunosuppression and none of our patients needed intraperitoneal displacement of the ureter after immunosuppression was included in the first line treatment. CRP values normalised in $55 \%$ of those patients in whom it had initially increased and improved in the others. Contrast enhancement improved in all patients with strong uptake.

The initial regimen consisted of cyclophosphamide (CYC) in nearly $50 \%$. Usually 6 (range $5-9$ ) intravenous CYC pulses were given (mean cumulative dose $5.9 \mathrm{~g}$, range 4.5-8.0) and patients were then switched to either azathioprine (AZA, 2$3 \mathrm{mg} / \mathrm{kg} /$ day) or oral corticosteroid (OCS) maintenance treatment. Eight patients received AZA. All of them responded, but three had to be switched because of new onset leucopenia or anaemia. Ciclosporin A was used twice and had to be discontinued in one patient because of new onset hypertension. Three patients (two receiving mycophenolate mofetil (MMF) and one receiving OCS monotherapy) progressed with the initial immunosuppression.

Five patients were treated only surgically. Aortic repair was performed in three patients with IAAA (patients 16, 17, and 20) and ureterolysis in two patients with isolated RPF (patients 4 and 12). One of the patients with IAAA received an intraluminal stent and two a synthetic graft. Two patients were lost to follow up and the third is in partial remission as measured by renal function and inflammatory variables. In both patients with isolated RPF, postoperative removal of DJ catheters is reported, but both were subsequently lost to follow up.

Two patients with IAAA received immunosuppressive treatment. One patient (patient 18) was treated postoperatively and went into full remission. In the other patient 
(No 19), who received initially successful OCS monotherapy, a renal artery obstruction had to be stented after 3 years. After intravenous CYC followed by AZA this patient is now in partial remission and receives low dose OCS alone.

\section{DISCUSSION}

We retrospectively examined the medical outcome of 20 patients with chronic periaortitis receiving surgical and/or immunosuppressive treatment.

As reported previously, chronic periaortitis was diagnosed mostly in the fifth decade of life,,$^{8-10}$ with non-specific and illdefined flank, back, or abdominal pain. ${ }^{8-10}$ Fever was uncommon.

Smoking has been recently described as a risk factor ${ }^{11}$ and was prevalent in $90 \%$ of our patients with IAAA and RPF. We were unable to assess other risk factors, such as asbestos exposure, ${ }^{11}$ in our retrospective analysis but noted a coincidence with other autoimmune disorders. ${ }^{72-14}$

There are no specific laboratory tests for chronic periaortitis. CRP or BSG are initially increased with very varying (and at times only very low) frequency. ${ }^{8}{ }^{10}{ }^{15}$ In our series, the CRP level was increased mostly in patients with strongly contrast enhancing lesions, but was not associated with the diameter of the periaortic mantle. Although the CRP often (but not unequivocally) normalised before the gadolinium uptake and the diameter of the periaortic mantle, normal CRP values did not exclude worsening imaging parameters. These facts suggest that the CRP is not a reliable marker for excluding chronic periaortitis and for monitoring disease activity.

Imaging has a major role in the diagnosis and should be performed in patients with unexplained back, flank, or abdominal pain. Although ultrasound is useful for detecting urinary tract obstruction or aortic aneurysms, it is limited in describing the extent of RPF, or in distinguishing between inflammatory and non-inflammatory AAA. ${ }^{16}$ Contrast enhanced MRI and CT permit more detailed imaging of chronic periaortitis, but it is unclear which technique is better. ${ }^{17}{ }^{18}$ MRI may be preferred because of its ability to obtain sagittal as well as coronal images, and because it avoids exposure to $x$ rays and iodinated contrast medium.

Since the first publication of successful treatment with OCS in $1958^{19}$ several reports have suggested that immunosuppressive treatment is effective. ${ }^{20}{ }^{21}$ In our study, disease activity was eventually controlled by immunosuppression in $13 / 15(87 \%)$ of patients, although modifications were often required. Mild to moderate cases were treated with AZA ( $2 \mathrm{mg} / \mathrm{kg}$ body weight) plus OCS, whereas severe or refractory disease was treated by monthly intravenous pulse of CYC ( $15 \mathrm{mg} / \mathrm{kg}$ body weight) for 6 months, followed by AZA. All patients treated with CYC went into remission and had no side effects. Overall, our experience and that of others $^{22-24}$ suggests that immunosuppressive agents have a beneficial effect. Only, MMF appeared to be less efficient in two of our patients than suggested in previous reports. ${ }^{25}$ Tamoxifen has also been used successfully on several occasions, ${ }^{26}$ but there are no data which allow a comparison of regimens.

In patients presenting with IAAA, the role of primary immunosuppression is less clear. Surgical repair of the aneurysm has been suggested as the preferred treatment, and seems to decrease the concomitant periaortal inflammation in about $50 \%$ of cases. ${ }^{15}{ }^{27}$ The indication for operative repair is the same as for non-inflammatory aneurysms, but the perioperative risk of septicaemia and renal failure may be higher. The role of additional ureterolysis is not yet settled, but the data seem to permit a careful observation of the spontaneous postoperative course after aneurysm repair. Preoperative steroids were, on the one hand, recommended to reduce the inflammatory mass ${ }^{28}$ and, on the other, advised against ${ }^{29}$ because of a presumed risk of intraoperative aortic rupture. Postoperative immunosuppression, however, may benefit some patients as suggested by our experience.

We conclude that immunosuppressive treatment is an effective and well tolerated treatment for RPF and should therefore be included in the early management of disease. Response to treatment may be protracted and should be monitored with imaging studies.

\section{ACKNOWLEDGEMENTS}

The study was performed within the framework of the German Rheumatology Network Centre, Freiburg, supported by BMBF grant No 01 GI9949 (HHP).

\section{Authors' affiliations}

K Warnatz, A G Keskin, C Scholz, P Vaith, H H Peter, U A Walker, Department of Rheumatology and Clinical Immunology, Albert-Ludwigs University, Freiburg, Germany

M Uhl, Department of Radiology, Albert-Ludwigs University, Freiburg,

Germany

A Katzenwadel, Department of Urology, Albert-Ludwigs University, Freiburg, Germany

\section{REFERENCES}

1 Ormond JK. Bilateral ureteral obstruction due to development and compression by an inflammatory process. J Urol 1948;59:1072-9.

2 Walker DI, Bloor K, Williams G, Gillie I. Inflammatory aneurysms of the abdominal aorta. Br J Surg 1972;59:609-14.

3 Mitchinson MJ. Chronic periaortitis and periarteritis. Histopathology 1984;8:589-600.

4 Gilkeson GS, Allen NB. Retroperitoneal fibrosis. A true connective tissue disease. Rheum Dis Clin North Am 1996;22:23-38.

5 Parums DV, Dunn DC, Dixon AK, Mitchinson MJ. Characterization of inflammatory cells in a patient with chronic periaortitis. Am J Cardiovasc Pathol 1990;3:121-9.

6 Parums DV, Brown DL, Mitchinson MJ. Serum antibodies to oxidized lowdensity lipoprotein and ceroid in chronic periaortitis. Arch Pathol Lab Med 1990; 114:383-7.

7 Izzedine H, Servais A, Launay-Vacher V, Deray G. Retroperitoneal fibrosis due to Wegener's granulomatosis: a misdiagnosis as tuberculosis. Am J Med 2002;113:164-6.

8 Wagenknecht LV, Auvert J. Symptoms and diagnosis of retroperitoneal fibrosis. Analysis of 31 cases. Urol Int 1971;26:185-95.

9 Baker LR, Mallinson WJ, Gregory MC, Menzies EA, Cattell WR, Whitfield HN, et al. Idiopathic retroperitoneal fibrosis. A retrospective analysis of 60 cases. Br J Urol 1987;60:497-503.

10 Koep L, Zuidema GD. The clinical significance of retroperitoneal fibrosis. Surgery 1977;81:250-7

11 Uibu T, Oksa P, Auvinen A, Honkanen E, Metsarinne K, Saha H, et al. Asbestos exposure as a risk factor for retroperitoneal fibrosis. Lancet 2004;363: 1422-6.

12 Vaglio A, Manenti L, Allegri L, Ferrozzi F, Corradi D, Buzio C. ANCA-positive periaortic vasculitis: does it fall within the spectrum of vasculitis? J Intern Med 2002;251:268-71.

13 Kaipiainen-Seppanen O, Jantunen E, Kuusisto J, Marin S. Retroperitoneal fibrosis with antineutrophil cytoplasmic antibodies. J Rheumatol 1996;23:779-81.

14 Okada H, Takahira S, Sugahara S, Nakamoto H, Suzuki H. Retroperitoneal fibrosis and systemic lupus erythematosus. Nephrol Dial Transplant 1999; 14:1300-2

15 Nitecki SS, Hallett JW Jr, Stanson AW, Ilstrup DM, Bower TC, Cherry KJ Jr, et al. nflammatory abdominal aortic aneurysms: a case-control study. J Vasc Surg 1996;23:860-8.

16 Tennant WG, Hartnell GG, Baird RN, Horrocks M. Radiologic investigation of abdominal aortic aneurysm disease: comparison of three modalities in staging and the detection of inflammatory change. J Vasc Surg 1993; 17:703-9.

17 Mulligan SA, Holley HC, Koehler RE, Koslin DB, Rubin E, Berland LL, et al. CT and MR imaging in the evaluation of retroperitoneal fibrosis. J Comput Assist Tomogr 1989;13:277-81.

18 Bachmann G, Baver T, Rau WS. MRI and CT in diagnosis and follow-up of idiopathic (retroperitoneal) fibrosis. Radiologe 1995;35:200-207.

19 Ross JC, Tinkler LF. Renal failure due to periureteric fibrosis. Br J Surg 1958;46:58-62.

20 Wagenknecht LV, Hardy JC. Value of various treatments for retroperitoneal fibrosis. Eur Urol 1981;7:193-200.

21 Marcolongo R, Tavolini IM, Laveder F, Busa M, Noventa F, Bassi P, et al. Immunosuppressive therapy for idiopathic retroperitoneal fibrosis: a retrospective analysis of 26 cases. Am J Med 2004;1 16:194-7.

22 Marzano A, Trapani A, Leone N, Actis GC, Rizzetto M. Treatment of idiopathic retroperitoneal fibrosis using cyclosporin. Ann Rheum Dis $2001 ; 60: 427-8$. 
23 Scavalli AS, Spadaro A, Riccieri V, Ricciuti GP, Taccari E, Marini M, et al. Long-term follow-up of low-dose methotrexate therapy in one case of idiopathic retroperitoneal fibrosis. Clin Rheumatol 1995; 14:481-4.

24 Kohler HP, Laeng RH, Egger C, Streuli R. Systemic fibrosis (generalized form of Ormond's disease). Report of a case which achieved complete remission with cyclophosphamide and corticosteroids. Schweiz Med Wochenschr 1995; 125:2131-6.

25 Grotz W, von Zl, Andre M, Schollmeyer P. Treatment of retroperitoneal fibrosis by mycophenolate mofetil and corticosteroids. Lancet 1998;352:1195.
26 Owens LV, Cance WG, Huth JF. Retroperitoneal fibrosis treated with tamoxifen. Am Surg 1995;61:842-4.

27 von Fritschen U, Malzfeld E, Clasen A, Kortmann H. Inflammatory abdominal aortic aneurysm: A postoperative course of retroperitoneal fibrosis. $J$ Vasc Surg 1999;30:1090-8.

28 Baskerville PA, Blakeney CG, Young AE, Browse NL. The diagnosis and treatment of peri-aortic fibrosis ('inflammatory' aneurysms). Br J Surg 1983;70:381-5

29 Gans RO, Hoorntje SJ, Rauwerda JA, Luth WJ, van Hattum LA, Donker AJ. The inflammatory abdominal aortic aneurysm. Prevalence, clinical features and diagnostic evaluation. Neth J Med 1993;43:105-15.

\section{Clinical Evidence - Call for contributors}

Clinical Evidence is a regularly updated evidence-based journal available worldwide both as a paper version and on the internet. Clinical Evidence needs to recruit a number of new contributors. Contributors are healthcare professionals or epidemiologists with experience in evidence-based medicine and the ability to write in a concise and structured way.

Areas for which we are currently seeking authors:

- Child health: nocturnal enuresis

- Eye disorders: bacterial conjunctivitis

- Male health: prostate cancer (metastatic)

- Women's health: pre-menstrual syndrome; pyelonephritis in non-pregnant women

However, we are always looking for others, so do not let this list discourage you.

Being a contributor involves:

- Selecting from a validated, screened search (performed by in-house Information Specialists) epidemiologically sound studies for inclusion.

- Documenting your decisions about which studies to include on an inclusion and exclusion form, which we keep on file.

- Writing the text to a highly structured template (about 1500-3000 words), using evidence from the final studies chosen, within 8-10 weeks of receiving the literature search.

- Working with Clinical Evidence editors to ensure that the final text meets epidemiological and style standards.

- Updating the text every six months using any new, sound evidence that becomes available. The Clinical Evidence in-house team will conduct the searches for contributors; your task is simply to filter out high quality studies and incorporate them in the existing text.

- To expand the topic to include a new question about once every 12-18 months.

If you would like to become a contributor for Clinical Evidence or require more information about what this involves please send your contact details and a copy of your CV, clearly stating the clinical area you are interested in, to Klara Brunnhuber (kbrunnhuber@ bmigroup.com).

\section{Call for peer reviewers}

Clinical Evidence also needs to recruit a number of new peer reviewers specifically with an interest in the clinical areas stated above, and also others related to general practice. Peer reviewers are healthcare professionals or epidemiologists with experience in evidence-based medicine. As a peer reviewer you would be asked for your views on the clinical relevance, validity, and accessibility of specific topics within the journal, and their usefulness to the intended audience (international generalists and healthcare professionals, possibly with limited statistical knowledge). Topics are usually 1500-3000 words in length and we would ask you to review between 2-5 topics per year. The peer review process takes place throughout the year, and our turnaround time for each review is ideally 10-14 days.

If you are interested in becoming a peer reviewer for Clinical Evidence, please complete the peer review questionnaire at www.clinicalevidence.com or contact Klara Brunnhuber (kbrunnhuber@bmigroup.com). 